Abstracted/indexed in Academic Search Complete, Asia Journals Online, Bangladesh Journals Online, Biological Abstracts, BIOSIS Previews, CAB Abstracts, Current Abstracts, Directory of Open Access Journals, EMBASE/Excerpta Medica, Google Scholar, HINARI (WHO), International Pharmaceutical Abstracts, Open J-gate, Science Citation Index Expanded, SCOPUS and Social Sciences Citation Index;

ISSN: $1991-0088$

\title{
Synthesis and preliminary evaluation of brominated 5-methyl-2,4- dihydropyrazol-3-one and its derivatives as cytotoxic agents
}

\author{
Rahat Khan, Md. Imam Uddin, Md. Sultan Alam, Mohammad Mamun Hossain and \\ Md. Rabiul Islam
}

Department of Chemistry, Jahangirnagar University, Savar, Dhaka 1342, Bangladesh.

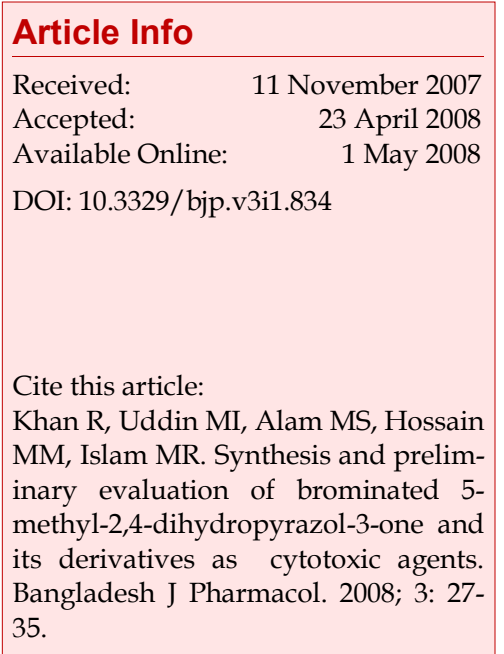

\begin{abstract}
Derivatives of 5-methyl-2,4-dihydro-pyrazol-3-one (1a), 5-methyl-2-phenyl2,4-dihydro-pyrazol-3-one (1b) and 2-(2,4-dinitro-phenyl)-5-methyl-2,4dihydro-pyrazol-3-one (1c) have been brominated in various ways. The compounds $(\mathbf{1} \mathbf{a}, \mathbf{1} \mathbf{b}, \mathbf{1 c})$ were synthesized by condensation reaction between the ethylacetoacetate and hydrazine derivatives. All the products have been characterized by extensive use of IR, ${ }^{1} \mathrm{H}-\mathrm{NMR},{ }^{13} \mathrm{C}-\mathrm{NMR}$ and Mass spectral analysis. Investigation of the cytotoxicity of these compounds was carried out against brine shrimp by lethality bioassay. The cytotoxicity studies of the synthesized compounds revealed that compound 4,4-dibromo-2-(2,4-dinitrophenyl)-5-methyl-2,4-dihydro-pyrazol-3-one (2c) had shown tremendous bioactivity against brine shrimp. However, the compounds 4,4-dibromo-5methyl-2,4-dihydro-pyrazol-3-one (2a), 4,4-dibromo-2-(2,4-dibromo-phenyl)-5 -methyl-2,4-dihydro-pyrazol-3-one (2b) and 4,4-dibromo-2-(dinitro-phenyl)-5methyl-2,4-dihydro-pyrazol-3-one (2c) showed higher activity leaving the other compounds almost inactive.
\end{abstract}

\section{Introduction}

Pyrazole chemically known as 1,2-diazole has become a popular topic due to its manifold uses. The chemistry of pyrazolone and its derivatives is particularly interesting because of their potential application in medicinal chemistry as analgesic (GÜrsoy et al., 2000; Amir et al., 2008), anti-inflammatory (Satyanarayana and Rao, 1995), antipyretic (Manna et al., 1992), antiparasitic (Kuettel et al., 2007), antimalarial (Kativar et al., 2005), antifungal (Bekhit and Fahmy, 2003) and enzyme inhibitory agents (Regan et al., 2003; Li et al., 2004 and Dolle et al., 1994). As an example, edaravone (3-methyl1-phenyl-2-pyrazolin-5-one, 1a) has been recently shown to produce marked attenuation of brain damage caused by ischemia-reperfusion (Anzai et al., 2004) and its pharmacological actions were attributed to its anti- oxidant activity, as a potent hydroxyl radical scavenger (Parmar et al., 1999). The useful properties of pyrazole derivatives as insecticides, fungicides and sedatives drew attention of many investigators. Earlier experiments showed that pyrazole derivatives can be used as blood platelet aggregation inhibitor. That is pyrazole and its derivatives are clearly important in the field of enzyme inhibition. Selvam et al. (2006) showed that brominated derivatives are strikingly cytotoxic and possess more bioactivity.

So, the present work is the continuation of bromination of 5-methyl-2,4-dihydro-pyrazol-3-one at its active methylene group (Islam et al., 2001). The bromination of 5-Methyl-2-phenyl-2,4-dihydro-pyrazol-3-one (1b) led to the expected bromination at active methylene group along with bromination at ortho- and para- 


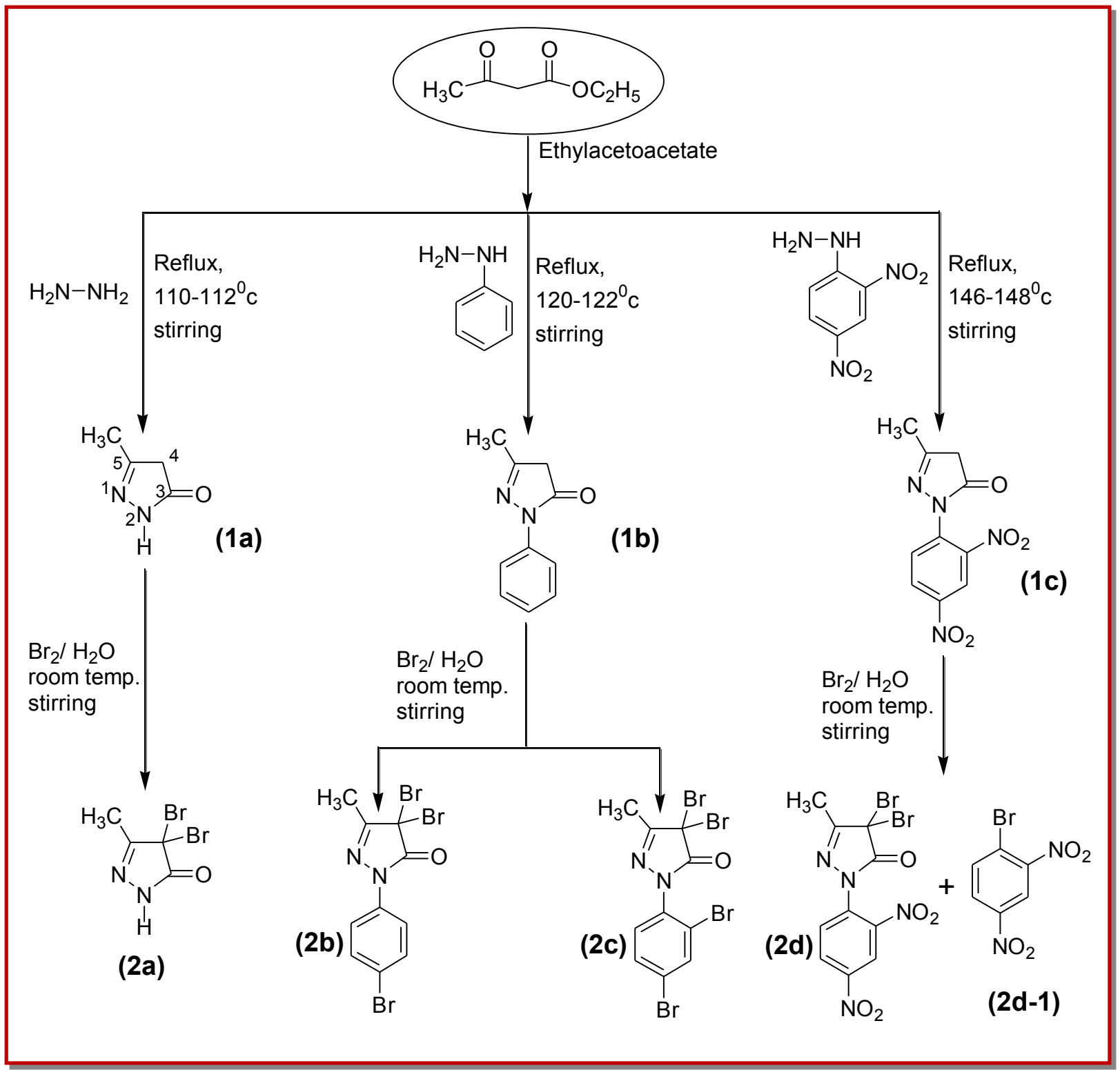

Scheme 1

position of the phenyl ring. This result is not consistent with the reported work of Elguero et al., 1984. So, the bromination on some of the pyrazoline-5-one derivatives (Scheme 1) was taken for investigation. Hence the results of bromination on the active methylene group of ethyl acetoacetate followed by the ring-closure reaction with hydrazine and hydrazine derivatives leading to the pyrazolone ring are reported along with its cytotoxicity studies.

\section{Materials and Methods}

The melting points of the synthesized compounds were recorded by thin disc method on a Fischer Jonhns electro -thermal melting point apparatus. Infrared spectra were recorded on DR-8001, Shimadzu FT-IR spectrophotometer, ${ }^{1} \mathrm{H}-\mathrm{NMR}$ spectra on a WP-400 NMR spectrophotometer using tetramethylsilane as internal standard. Mass spectra were obtained on a Kratas MS-25 using DH-88 data system.

Three active methylene compounds (1a-c) were prepared by condensation of ethylacetoacetate and hydrazine and its derivatives in moderate yields (Scheme 1). Bromination was carried out on these three products (1a-c) by following the standard procedure of Elguero et al., 1984 and dibromo (2a and 2d), tribromo (2d), tetrabromo $(2 \mathrm{c})$ along with a side product $(2 \mathrm{~d}-1)$ were obtained. Compound $2 \mathrm{~d}$ was also prepared by an alternative method as shown in Scheme 2. Here bromination was carried out first on ethylacetoacetate to get 


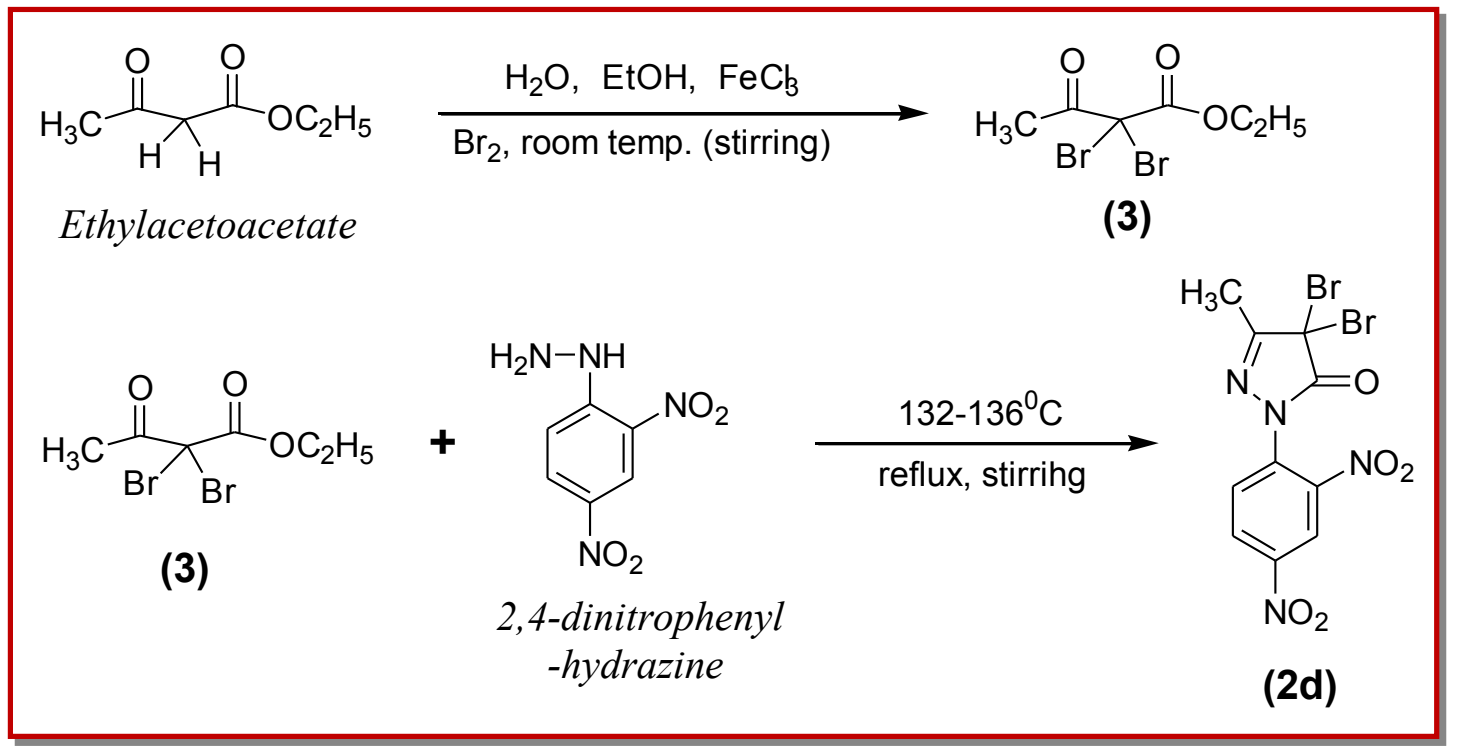

Scheme 2

compound 3. This compound on condensation with 2,4dinitrophenyl hydrazine at $132-136^{\circ} \mathrm{C}$ afforded $2 \mathrm{~d}$ in high yield. Compound (2e) was obtained from (1c) by bromination with NBS.

General procedure for the preparation of 5-methyl-2,4dihydro-pyrazol-3-ones, (1a-c)

Ethylacetoacetate was taken in a around bottomed flask and hydrazine hydrate and its derivatives were added to the flask dropwise with constant stirring at room temperature whereupon a vigorous reaction set in. A precipitate was formed quickly. As the reaction was exothermic, the reaction mixture was allowed to cool at room temperature. The resulting precipitate was collected by filtration. The crude product $(1.5 \mathrm{~g})$ was purified by recrystalization. The products were identified by spectroscopic techniques. General procedure for the preparation of 4,4-dibromo-5
-methyl-2,4-dihydro-pyrazol-3-ones, $(2 a-2 d)$

Compound 1a-1c were taken in a round-bottomed flask and dissolved in water $\left(300 \mathrm{~cm}^{3}\right)$. Bromine $\left(15 \mathrm{~cm}^{3}\right)$ was added to the solution from dropping funnel whereupon a vigorous reaction set in. After hydrogen bromide had ceased to evolve, the solid product was isolated by suction, washed with water several times and recrystallized from petroleum ether $\left(60-80^{\circ} \mathrm{C}\right)$ to give crystals of $\mathbf{2 a - 2 d}$. During the Bromination of $\mathbf{1 c}$ an unexpected byproduct was found from column chromatographic separation as the second fraction which lowers the $\%$ of yield of our main product, $2 \mathrm{~d}$. The products were identified by spectroscopic techniques.

Preparation of 2,2-dibromo-3-oxo-butyric acid ethyl ester, 3

Ethylacetoacetate $(14.5123 \mathrm{~g}, 0.1209 \mathrm{mmol})$ was taken in a two-necked round bottomed flask and dissolved in ethanol (15 mL). 2-3 drops of aqueous solution of $\mathrm{FeCl}_{3}$ was added to the stirred solution which produced deep violet-red coloration. $100 \mathrm{~mL}$ of water was added to it with constant stirring. To this solution $\mathrm{Br}_{2} / \mathrm{H}_{2} \mathrm{O}$ was added from a dropping funnel until the deep violet-red color was just disappeared. Upon standing the deep violet-red color was reappeared. This process was continued until the deep red-violet coloration was disappeared permanently. Some excess amount of $\mathrm{Br}_{2} /$ $\mathrm{H}_{2} \mathrm{O}$ was added with constant stirring for 0.5 hour. Then the excess amount of $\mathrm{Br}_{2}$ was removed by adding $5 \%$ aqueous solution of $\mathrm{NaHSO}_{3}$. Compound 3 was extracted by chloroform and was pale yellow colored liquid (Scheme 2).

Preparation of 4,4-dibromo-2-(dinitro-phenyl)-5methyl-2,4-dihydro-pyrazol-3-one, $2 d$

A mixture of compound $3(0.0500 \mathrm{~g}, 0.1737 \mathrm{mmol})$ and an equivalent amount of 2,4-dinitrophenyl hydrazine $(0.0344 \mathrm{~g}, 0.1737 \mathrm{mmol})$ was taken in a $100 \mathrm{~mL}$ two necked quick-fit round-bottomed flask and was refluxed in an oil-bath for 3.5 hours. The color of the reaction mixture was changed to deep red during the reflux period. The reaction mixture was allowed to cool at room temperature and was kept overnight to be precipitated out. Then the reaction mixture was treated with water, immediately brown solid came out. The crude product was purified by recrystalization from chloroform that gave brown crystalline solid of 2c; m.p. $120-121^{\circ} \mathrm{C}$; yield $0.0641 \mathrm{~g}(88 \%), \mathrm{R}_{\mathrm{f}} 0.65$ (PE:EA, 4:1). The product was characterized spectroscopically (Scheme 


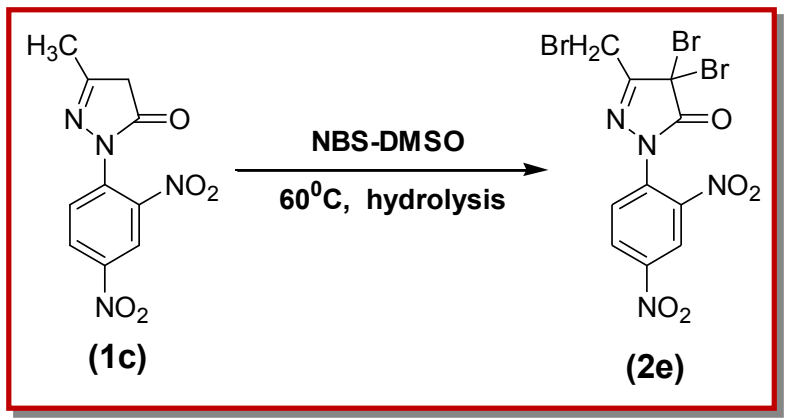

Scheme 3

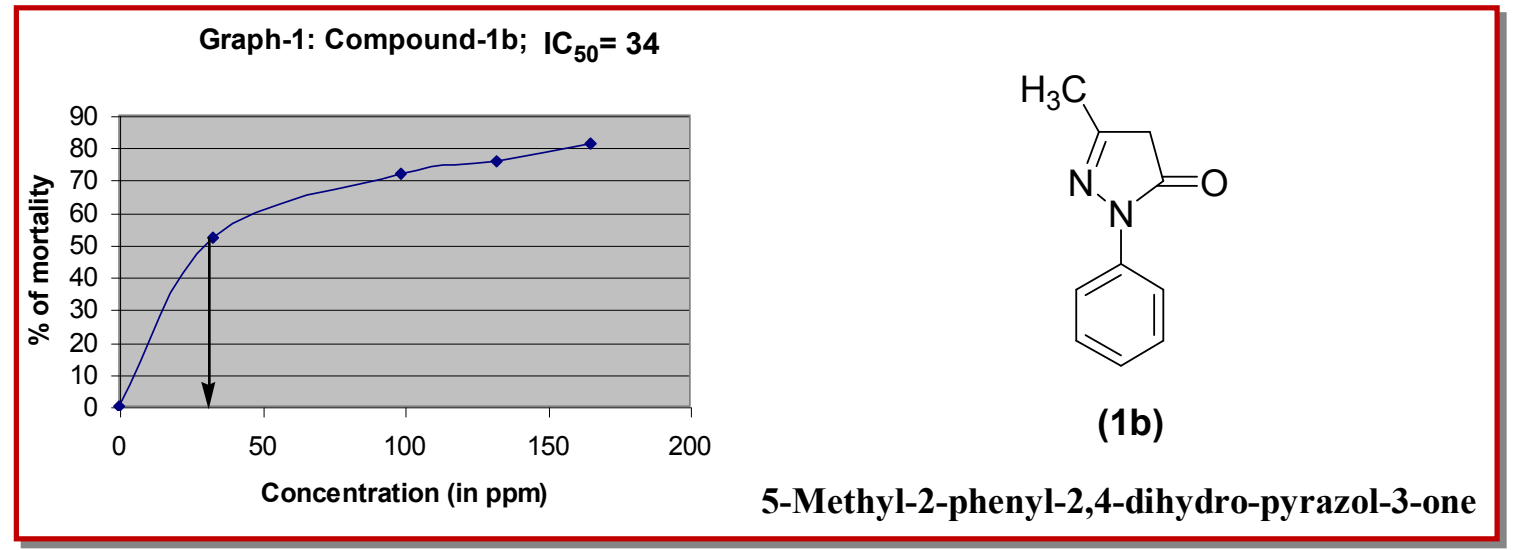

Figure 1: Graph that was used for determining the $\mathrm{IC}_{50}$ of compound $1 \mathrm{~b}$

2).

Preparation of 4,4-dibromo-5-bromomethyl-2-(2,4dinitrophenyl)-2,4-dihydro-pyrazol-3-one, $2 e$

N-bromosuccinimide, NBS (3.57 g, $0.0201 \mathrm{~mol})$ in anhydrous DMSO (15 mL) was added slowly to the stirred solution of the compound 1c (7.3495 g, 0.0278 $\mathrm{mol})$ in anhydrous DMSO $(5 \mathrm{ml})$ under an inert atmosphere. The solution was stirred at $60^{\circ} \mathrm{C}$ for 6 hours under an ambient pressure to give a red solution. After hydrogen bromide had ceased to evolve, the red solution was hydrolyzed with water, whereupon the solid product isolated. After hydrolysis the compound $2 \mathbf{e}$ was isolated by filtration and washed several times with water and dried by suction (Scheme 3). The crude product was purified by recrystalization from dichloromethane that gave red powdered solid of $\mathbf{2 e}$. The product was characterized spectroscopically.

\section{Demonstration of cytotoxicity}

The cytotoxicity study of the synthesized compounds 1a, 1b, 1c, 2a, 2b, 2c, 2d, 2d-1, 2e and 3 was investigated on brine shrimp as a test organism for convenience (Solis et al., 1993). $1.6 \mathrm{mg}$ of each of the compounds was taken in the corresponding sample vials with $1.6 \mathrm{~mL}$ of dimethyl sulfoxide to prepare stock solution. From this stock solution 33, 99, 132 and 165 ppm of each compounds were placed in separate test tubes by micro syringe $1 \mathrm{~mL}$ of extra dimethyl sulfoxide was given in each test tube with 10-12 brine shrimp. After 1, 2, 3 and 4 hours the test tubes were observed and the number of survived naupli in each test tube was counted and results were noted. From this the percentage of lethality of brine shrimp naupli was calculated at each concentration for each sample. Then graphs are drawn by plotting percentage of lethality of brine shrimp versus doses (in ppm) of the synthesized compounds which gave rise to the $\mathrm{IC}_{50}$ (inhibitory concentration-50) values of the corresponding compounds (Figure 1). $\mathrm{IC}_{50}$ of an agent is the dose which will inactive $50 \%$ of the test animal.

\section{Results}

The formations of the new compounds were ensured by its several physical constants, such as melting temperature $\left({ }^{\circ} \mathrm{C}\right), \mathrm{R}_{\mathrm{f}}$ values and color. However, the introduction of bromine atoms into the pyrazolone ring was identified by chemical method. Finally, the structures of the compounds were determined by the spectroscopic methods.

Physical constants gave the preliminary idea about the formation of new compounds. These constants are given here as a tabular form (Table I). 


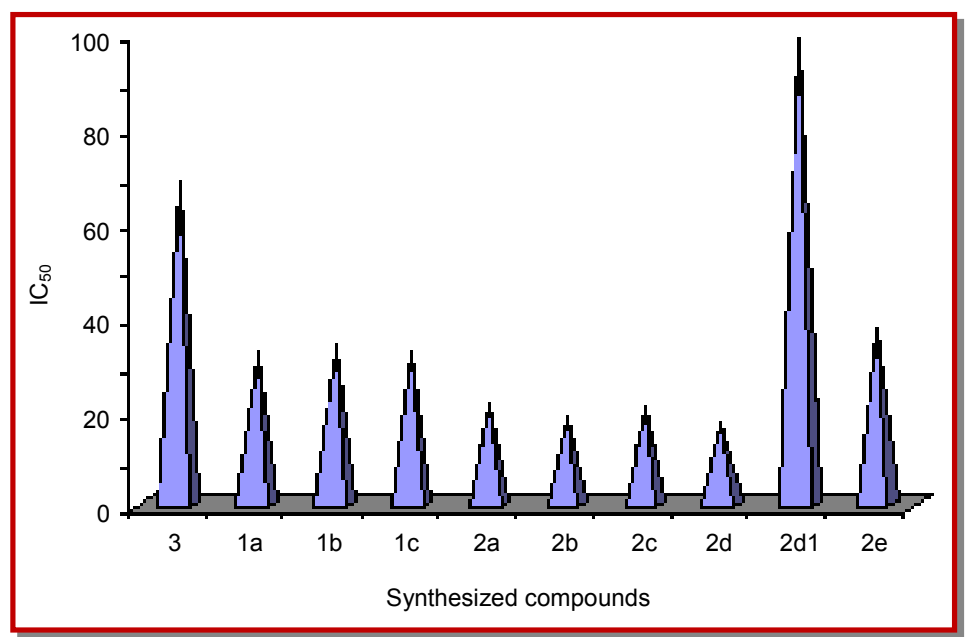

Figure 2: Graphical representation of $\mathrm{IC}_{50}$ of the synthesized compounds

\section{Table I}

Some physical data of the synthesized compounds

\begin{tabular}{|c|c|c|c|c|}
\hline Compounds & Melting points $\left({ }^{\circ} \mathrm{C}\right)$ & $\%$ of yield & $\mathrm{R}_{\mathrm{f}}$ values (PE:EA=1:2) & Color \\
\hline $1 \mathrm{a}$ & 223 & 30 & 0.32 & White \\
\hline $1 b$ & 127 & 78 & 0.52 & Pale yellow \\
\hline 1c & 81 & 98 & 0.74 & Yellow \\
\hline $2 a$ & 131 & 64 & 0.41 & White \\
\hline $2 b$ & 78 & 76 & 0.29 & Pale yellow \\
\hline 2c & 134 & 72 & 0.35 & Pale yellow \\
\hline $2 d$ & 120 & 49 & 0.65 & Dark red \\
\hline $2 d-1$ & 92 & 48 & 0.62 & Light yellow \\
\hline $2 e$ & 123 & 87 & 0.57 & Yellow \\
\hline 3 & 113 (b.p) & 97 & 0.68 & Yellow \\
\hline
\end{tabular}

Lassaigene test was performed on each of the synthesized compounds to detect the upcoming Bromine atoms. The results of the chemical tests are given bellow (Table II): The resulting spectroscopic datas are given bellow:

Compound 1a: IR (Nujol): 3480 (vN-H); 2980, 2880 (vC-H aliphatic); 2700 (vo-H enolic); 1680 ( $\mathrm{v}_{\mathrm{C}=\mathrm{O}}$ lactam); 1615 $\left(\mathrm{v}_{\mathrm{C}=\mathrm{N}}\right) ; 1580\left(\mathrm{v}_{\mathrm{C}=\mathrm{C}}\right.$ aromatic); $1515\left(\delta_{\mathrm{N}-\mathrm{H}}\right) \mathrm{cm}^{-1} ;{ }^{1} \mathrm{H}-\mathrm{NMR}$ $\left(\mathrm{DMSO}_{\mathrm{d}}\right)$ ) $\delta 5.2(\mathrm{~s}, 1 \mathrm{H},=\mathrm{CH}) ; 2.1\left(\mathrm{~s}, 3 \mathrm{H}, \mathrm{CH}_{3}\right) ; 10.0$ (s, $1 \mathrm{H}, \mathrm{NH}) ; 10.5$ (s, 1H, OH); ${ }^{13} \mathrm{C}-\mathrm{NMR}$ (DMSO-d 6 ): $\delta 11.4$ $\left(\mathrm{CH}_{3}\right), 89.23$ (C-4); 139.82 (C-3); 161.42 (=C-OH); MS: $m /$ $z$ (\% of rel. intensities): $98\left(\mathrm{M}^{+}, 100\right), 97(17), 81(1.0), 73$ (1.0), 67(18).

Compound 1b: IR (Nujol): 2980, 2880 (v $\mathrm{v}_{\mathrm{C}-\mathrm{H}}$ aliphatic); 2700 ( $\mathrm{v}_{\mathrm{O}-\mathrm{H}}$ enolic); 1697.7 ( $\mathrm{v}_{\mathrm{C}=\mathrm{O}}$ lactam); 1489.2, 1541.3 $\left(\mathrm{v}_{\mathrm{C}=\mathrm{N}}\right) ; 1616.5,1558.8\left(\mathrm{v}_{\mathrm{C}=\mathrm{C}}\right.$ aromatic); $1311.5\left(\mathrm{v}_{\mathrm{C}-\mathrm{N}}\right) ; 773.5$ $\left(\delta_{\mathrm{C}-\mathrm{H}}\right) \mathrm{cm}^{-1} ;{ }^{1} \mathrm{H}-\mathrm{NMR}\left(\mathrm{CDCl}_{3}\right): \delta 11.45$ (br, s, $\left.1 \mathrm{H}, \mathrm{OH}\right)$; $5.36(\mathrm{~s}, 1 \mathrm{H},=\mathrm{CH}) ; 2.1\left(\mathrm{~s}, 3 \mathrm{H}, \mathrm{CH}_{3}\right) ; 7.43(\mathrm{dd}, 2 \mathrm{H}$,
$\left.J_{m}=1.1 \mathrm{~Hz}, J_{o}=8.0 \mathrm{~Hz}\right) ; 7.71\left(\mathrm{dd}, 2 \mathrm{H}, J_{0}=7.4 \mathrm{~Hz}, J_{m}=1.1 \mathrm{~Hz}\right)$; $7.52\left(\mathrm{dd}, 1 \mathrm{H}, J_{m}=2.0 \mathrm{~Hz}, J_{o}=7.2 \mathrm{~Hz}\right) ;{ }^{13} \mathrm{C}-\mathrm{NMR}\left(\mathrm{CDCl}_{3}\right): \delta$ $14.60\left(\mathrm{CH}_{3}\right), 178.3(=\mathrm{C}-\mathrm{OH}) ; 45.10\left(\mathrm{CH}_{2}\right) ; 132.1(\mathrm{~N}=\mathrm{C})$; 147.0 (aromatic C-1); 132.01 (aromatic C-2/6); 142.30 (aromatic C-3/5); 130 (aromatic C-4).

Compound 1c: IR (KBr): 3116 (vC-H aromatic); 2925 (vC-H saturated); 1728 ( $\mathrm{v}_{\mathrm{C}=\mathrm{O}}$ lactam); $1620\left(\mathrm{v}_{\mathrm{C}=\mathrm{N}}\right) ; 1597,1473$ $\left(\left(\mathrm{v}_{\mathrm{C}=\mathrm{C}}\right.\right.$ aromatic); 1410, $1518\left(\left(\mathrm{v}_{\mathrm{N}=\mathrm{O}}\right.\right.$ nitro group); 1340 ( $\mathrm{v}_{\mathrm{C}}$ -N); $1026\left(\mathrm{v}_{\mathrm{C}-\mathrm{O}}\right) ; 1455,1378\left(\delta_{\mathrm{C}-\mathrm{H}}\right) ; 1357\left(\mathrm{v}_{\mathrm{N}-\mathrm{N}}\right) \mathrm{cm}^{-1} ;{ }^{1} \mathrm{H}-$ NMR (DMSO-d $)$ ): $\delta 10.85$ (br, s, 1H, OH); 3.61 (s, 2H, $\left.\mathrm{CH}_{2}\right) ; 2.19\left(\mathrm{~s}, 3 \mathrm{H}, \mathrm{CH}_{3}\right) ; 8.92\left(\mathrm{~d}, \mathrm{H}, J_{m}=3.2 \mathrm{~Hz}\right) ; 8.43(\mathrm{dd}$, $\left.1 \mathrm{H}, J_{o}=5.3 \mathrm{~Hz}, J_{m}=2.5 \mathrm{~Hz}\right) ; 7.86\left(\mathrm{dd}, 1 \mathrm{H}, J_{0}=7.6 \mathrm{~Hz}\right) ;{ }^{13} \mathrm{C}-$ $\operatorname{NMR}\left(\mathrm{CDCl}_{3}\right): \delta 16.525\left(\mathrm{CH}_{3}\right), 169.45(=\mathrm{C}-\mathrm{OH}) ; 61.03$ $\left(\mathrm{CH}_{2}\right) ; 153.54(\mathrm{~N}=\mathrm{C}) ; 116.30$ (aromatic C-6); 123.31 (aromatic C-1); 129.81 (aromatic C-2); 130.54 (aromatic C-4); 137.52 (aromatic C-5); 144.88 (aromatic C-3). MS: $\mathrm{m} / \mathrm{z}$ (\% of rel. intensities): $264\left(\mathrm{M}^{+}\right), 137(18 \%), 219(28 \%)$, $173(6 \%), 145(8 \%), 131$ (6\%), 104 (14\%), 77 (32\%), 51 


\begin{tabular}{|c|c|c|}
\hline \multicolumn{3}{|c|}{ Table II } \\
\hline \multicolumn{3}{|c|}{ Results of the chemical tests } \\
\hline Compounds & Nitrogen Present & Bromine Present \\
\hline $1 \mathrm{a}$ & $+v e$ & $-v e$ \\
\hline $1 b$ & $+v e$ & $-v e$ \\
\hline $1 c$ & $+v e$ & $-v e$ \\
\hline $2 a$ & $+v e$ & $+v e$ \\
\hline $2 b$ & $+v e$ & $+v e$ \\
\hline $2 c$ & $+v e$ & $+v e$ \\
\hline $2 d$ & $+v e$ & $+v e$ \\
\hline $2 d-1$ & $+v e$ & $+v e$ \\
\hline $2 \mathrm{e}$ & $+v e$ & $+v e$ \\
\hline 3 & $-v e$ & $+v e$ \\
\hline
\end{tabular}

Table III

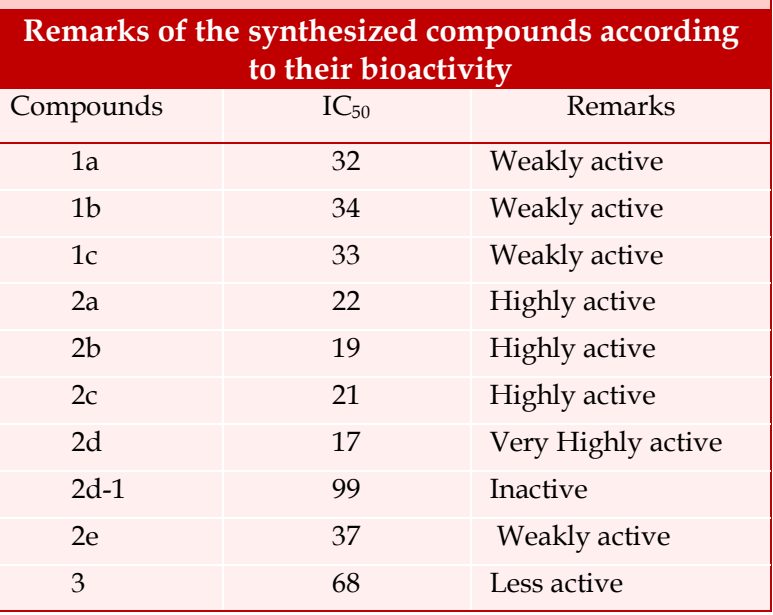

(8\%); Elemental analysis: Clalc. for $\left[\mathrm{C}_{10} \mathrm{H}_{8} \mathrm{~N}_{4} \mathrm{O}_{5}\right]$ : $\mathrm{C}$ $(46.48 \%), \mathrm{H}(4.276 \%), \mathrm{N}(18.43 \%)$. Found: C (46.41\%), H $(4.241 \%), \mathrm{N}(18.36 \%)$.

Compound 2a: IR (Nujol): $3353 \quad\left(\mathrm{v}_{\mathrm{N}-\mathrm{H}}\right) ; 2880 \quad\left(\mathrm{v}_{\mathrm{C}-\mathrm{H}}\right.$ aliphatic); 1723 ( $\mathrm{v}_{\mathrm{C}=\mathrm{O}}$ lactam); $1620\left(\mathrm{v}_{\mathrm{C}=\mathrm{N}}\right) ; 1300\left(\mathrm{v}_{\mathrm{C}-\mathrm{N}}\right)$;

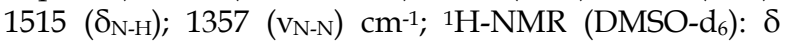
12.0 (s, 1H, NH); $2.1\left(\mathrm{~s}, 3 \mathrm{H}, \mathrm{CH}_{3}\right) ; \mathrm{MS}: \mathrm{m} / z$ (\% of rel. intensities): 254, 256, 258 (M, M+2, M+4, 10:20:10); 177/175 (100); 98 (3.0); 94 (2.0).

Compound 2b: IR (Nujol): 3094 (vC-H aromatic); 2916 (vC$\mathrm{H}$ aliphatic); 1738 ( $\mathrm{v}_{\mathrm{C}=\mathrm{O}}$ lactam); $1574\left(\mathrm{v}_{\mathrm{C}=\mathrm{N}}\right) ; 1616.5$, $1558.8 \quad\left(\mathrm{v}_{\mathrm{C}=\mathrm{C}}\right.$ aromatic); $1279 \quad\left(\mathrm{v}_{\mathrm{C}-\mathrm{N}}\right) ; 773.5 \quad\left(\delta_{\mathrm{C}-\mathrm{H}} p-\right.$ substituted benzene ring); 650 ( $\left.\mathrm{v}_{\mathrm{C}-\mathrm{Br}}\right) ; \mathrm{cm}^{-1} ;{ }^{1} \mathrm{H}-\mathrm{NMR}$ (DMSO-d 6$): \delta 2.3\left(\mathrm{~s}, 3 \mathrm{H}, \mathrm{CH}_{3}\right) ; 8.15\left(\mathrm{~d}, 1 \mathrm{H}, J_{m}=2.26 \mathrm{~Hz}\right)$; $7.77\left(\mathrm{dd}, 1 \mathrm{H}, J_{o}=8.54 \mathrm{~Hz}, J_{m}=2.26 \mathrm{~Hz}\right) ; 7.52(\mathrm{~d}, 1 \mathrm{H}$, $J_{o}=8.54 \mathrm{~Hz}$ ). MS: $m / z$ (\% of rel. intensities): 486, 488, 490, 492, $494\left(\mathrm{M}^{+}, \mathrm{M}+2, \mathrm{M}+4, \mathrm{M}+6, \mathrm{M}+8,1: 4: 6: 4: 1\right) ; 408,410$, 412 , $414\left(\mathrm{M}^{+}-\mathrm{Br}, 1: 3: 3: 1\right) ; 488(100 \%) ; 329(20 \%) ; 289(5 \%)$;
$263(50 \%) ; 235(65 \%) ; 182(5 \%) ; 154(18 \%) ; 117(5 \%) ; 75$ $(20 \%)$.

Compound 2c: IR (KBr): 3118 ( $\mathrm{v}_{\mathrm{C}-\mathrm{H}}$ aromatic); 2927 ( $\mathrm{v}_{\mathrm{C}-\mathrm{H}}$ saturated); 1714 ( $\mathrm{v}_{\mathrm{C}=\mathrm{O}}$ lactam); $1612\left(\mathrm{v}_{\mathrm{C}=\mathrm{N}}\right) ; 1593,1498$ $\left(\left(\mathrm{v}_{\mathrm{C}=\mathrm{C}}\right.\right.$ aromatic $) ; 1427,1548\left(\left(\mathrm{v}_{\mathrm{N}=\mathrm{O}}\right.\right.$ nitro group $) ; 1338\left(\mathrm{v}_{\mathrm{C}}\right.$ -N); $1026\left(\mathrm{v}_{\mathrm{C}-\mathrm{O}}\right) ; 1455,1315\left(\delta_{\mathrm{C}-\mathrm{H}}\right) ; 1357\left(\mathrm{v}_{\mathrm{N}-\mathrm{N}}\right) ; 742$ ( $\left.\mathrm{v}_{\mathrm{C}-\mathrm{Br}}\right)$; $\mathrm{cm}^{-1} ;{ }^{1} \mathrm{H}-\mathrm{NMR}\left(\mathrm{CDCl}_{3}\right): \delta 1.55\left(\mathrm{~s}, 3 \mathrm{H}, \mathrm{CH}_{3}\right) ; 8.71(\mathrm{~d}, \mathrm{H}$, $\left.J_{m}=2.5 \mathrm{~Hz}\right) ; 8.30\left(\mathrm{dd}, 1 \mathrm{H}, J_{o}=8.7 \mathrm{~Hz}, J_{m}=2.5 \mathrm{~Hz}\right) ; 8.01(\mathrm{~d}$, $\left.1 \mathrm{H}, J_{0}=8.07 \mathrm{~Hz}\right) ;{ }^{13} \mathrm{C}-\mathrm{NMR}\left(\mathrm{CDCl}_{3}\right): \delta 14.28\left(\mathrm{CH}_{3}\right), 190.20$ $(\mathrm{C}=\mathrm{O}) ; 107.20\left(\mathrm{CBr}_{2}\right) ; 187.11(\mathrm{~N}=\mathrm{C}) ; 120.97$ (aromatic $\mathrm{C}$ 6); 121.02 (aromatic C-1); 128.08 (aromatic C-2); 136.56 (aromatic C-4); 147.31 (aromatic C-5); 150.08 (aromatic C-3). MS: $m / z$ (\% of rel. intensities): $420,422,424\left(\mathrm{M}^{+}\right.$, $\mathrm{M}+2, \mathrm{M}+4,1: 2: 1) ; 281,283(1: 1,1.5 \%) ; 246,248$ (1:1, 100\%); 167 (3.99\%), 156 (53.8\%), 154 (23.7\%), 75 $(93.41 \%)$; Elemental analysis: Clalc. for $\left[\mathrm{C}_{10} \mathrm{H}_{6} \mathrm{Br}_{2} \mathrm{~N}_{4} \mathrm{O}_{5}\right]: \mathrm{C}$ $(28346 \%), \mathrm{H}(1.43 \%), \mathrm{N}(13.28 \%)$. Found: C $(28.45 \%), \mathrm{H}$ $(1.46 \%), \mathrm{N}(13.26 \%)$.

Compound 2d: IR (KBr): 3118 ( $\mathrm{v}_{\mathrm{C}-\mathrm{H}}$ aromatic); 2927 ( $\mathrm{v}_{\mathrm{C}-\mathrm{H}}$ saturated); 1714 ( $\mathrm{v}_{\mathrm{C}=\mathrm{O}}$ lactam); $1612\left(\mathrm{v}_{\mathrm{C}=\mathrm{N}}\right) ; 1593,1498$ $\left(\left(\mathrm{v}_{\mathrm{C}=\mathrm{C}}\right.\right.$ aromatic); 1427, $1548\left(\left(\mathrm{v}_{\mathrm{N}=\mathrm{O}}\right.\right.$ nitro group $) ; 1338\left(\mathrm{v}_{\mathrm{C}}\right.$ -N); $1026\left(\mathrm{v}_{\mathrm{C}-\mathrm{O}}\right) ; 1455,1315\left(\delta_{\mathrm{C}-\mathrm{H}}\right) ; 1357\left(\mathrm{v}_{\mathrm{N}-\mathrm{N}}\right) ; 742\left(\mathrm{v}_{\mathrm{C}-\mathrm{Br}}\right)$; $\mathrm{cm}^{-1} ;{ }^{1} \mathrm{H}-\mathrm{NMR}\left(\mathrm{CDCl}_{3}\right): \delta 1.55\left(\mathrm{~s}, 3 \mathrm{H}, \mathrm{CH}_{3}\right) ; 8.71(\mathrm{~d}, \mathrm{H}$, $\left.J_{m}=2.5 \mathrm{~Hz}\right) ; 8.30\left(\mathrm{dd}, 1 \mathrm{H}, J_{o}=8.7 \mathrm{~Hz}, J_{m}=2.5 \mathrm{~Hz}\right) ; 8.01(\mathrm{~d}$, $\left.1 \mathrm{H}, J_{0}=8.07 \mathrm{~Hz}\right) ;{ }^{13} \mathrm{C}-\mathrm{NMR}\left(\mathrm{CDCl}_{3}\right): \delta 14.28\left(\mathrm{CH}_{3}\right), 190.20$ $(\mathrm{C}=\mathrm{O}) ; 107.20\left(\mathrm{CBr}_{2}\right) ; 187.11(\mathrm{~N}=\mathrm{C}) ; 120.97$ (aromatic $\mathrm{C}$ 6); 121.02 (aromatic C-1); 128.08 (aromatic C-2); 136.56 (aromatic C-4); 147.31 (aromatic C-5); 150.08 (aromatic C-3). MS: $m / z$ (\% of rel. intensities): 420, 422, $424\left(\mathrm{M}^{+}\right.$, $\mathrm{M}+2$, M+4, 1:2:1); 281,283 (1:1, 1.5\%); 246, 248 (1:1, 100\%); 167 (3.99\%), 156 (53.8\%), 154 (23.7\%), 75 (93.41\%); Elemental analysis: Clalc. for $\left[\mathrm{C}_{10} \mathrm{H}_{6} \mathrm{Br}_{2} \mathrm{~N}_{4} \mathrm{O}_{5}\right]: \mathrm{C}$ $(28346 \%), \mathrm{H}(1.43 \%), \mathrm{N}(13.28 \%)$. Found: C $(28.45 \%), \mathrm{H}$ $(1.46 \%), \mathrm{N}(13.26 \%)$.

Compound 2d-1: IR (KBr): 3118 ( $\mathrm{v}_{\mathrm{C}-\mathrm{H}}$ aromatic); 1593, 1498 (( $\mathrm{v}_{\mathrm{C}=\mathrm{C}}$ aromatic); 1427, 1548 (( $\mathrm{v}_{\mathrm{N}=\mathrm{O}}$ nitro group); 1338 ( $\left.\mathrm{v}_{\mathrm{C}-\mathrm{N}}\right) ; 742\left(\mathrm{v}_{\mathrm{C}-\mathrm{Br}}\right) ; \mathrm{cm}^{-1} ;{ }^{1} \mathrm{H}-\mathrm{NMR}\left(\mathrm{CDCl}_{3}\right): \delta 8.84(\mathrm{~d}$, $\left.\mathrm{H}, J_{m}=2.39 \mathrm{~Hz}\right) ; 8.38\left(\mathrm{dd}, 1 \mathrm{H}, J_{o}=8.7 \mathrm{~Hz}, J_{m}=2.39 \mathrm{~Hz}\right) ; 8.21$ (d, $\left.1 \mathrm{H}, J_{o}=8.79 \mathrm{~Hz}\right)$. MS: $m / z$ (\% of rel. intensities): $246 / 248$ (M+/ M+2, 11\%/10\%); 216/218 (5\%); 200/202 (3.60\%, 3.27\%); $170 / 172(4.64 \%, 3.80 \%) ; 167$ (3.02\%); $154 / 156 \quad(6.2 \%, 7.32 \%) ; 134 \quad(100 \%) ; 120(2.95 \%) ; 91$ (6.76\%); 90 (3.29\%); 75 (15.02\%); 74 (3.84\%).

Compound 2e: IR (KBr): 3030 ( $\mathrm{v}_{\mathrm{C}-\mathrm{H}}$ aromatic); 2926 ( $\mathrm{v}_{\mathrm{C}-\mathrm{H}}$ saturated); $1684(\mathrm{vC}=\mathrm{O}$ lactam $) ; 1620\left(\mathrm{v}_{\mathrm{C}=\mathrm{N}}\right) ; 1549,1498$ $\left(\left(\mathrm{v}_{\mathrm{C}=\mathrm{C}}\right.\right.$ aromatic); 1376, $1538\left(\left(\mathrm{v}_{\mathrm{N}=\mathrm{O}}\right.\right.$ nitro group $) ; 1318\left(\mathrm{v}_{\mathrm{C}}\right.$ $\left.{ }_{-\mathrm{N}}\right) ; 1445,1315\left(\mathrm{\delta}_{\mathrm{C}-\mathrm{H}}\right) ; 744\left(\mathrm{v}_{\mathrm{C}-\mathrm{Br}}\right) ; \mathrm{cm}^{-1} ;{ }^{1} \mathrm{H}-\mathrm{NMR}\left(\mathrm{CDCl}_{3}\right)$ : ठ $2.44\left(\mathrm{~s}, 2 \mathrm{H}, \mathrm{CH}_{2}\right) ; 8.84\left(\mathrm{~d}, 1 \mathrm{H}, J_{m}=2.79 \mathrm{~Hz}\right) ; 8.379(\mathrm{dd}$, $\left.1 \mathrm{H}, J_{o}=8.79 \mathrm{~Hz}, J_{m}=2.79 \mathrm{~Hz}\right) ; 8.20\left(\mathrm{~d}, 1 \mathrm{H}, J_{o}=8.79 \mathrm{~Hz}\right)$. MS: $m / z$ (\% of rel. intensities): 498/500/502/504 (M+, M+2 , $\mathrm{M}+4, \quad \mathrm{M}+6, \quad 29.24 \%) ; \quad 458 / 460 / 462 / 464 \quad(59.76 \%)$; $418 / 420 / 422 \quad(8.92 \%) ; \quad 338 / 340 \quad(8.53 \%)$; $292 / 298 / 296 / 298 \quad(100 \%) ; \quad 277 / 279 \quad(64.88 \%)$; $264 / 266 / 268 / 270 \quad(11.81 \%) ; 251 / 253 / 255 \quad(30.84 \%)$; 


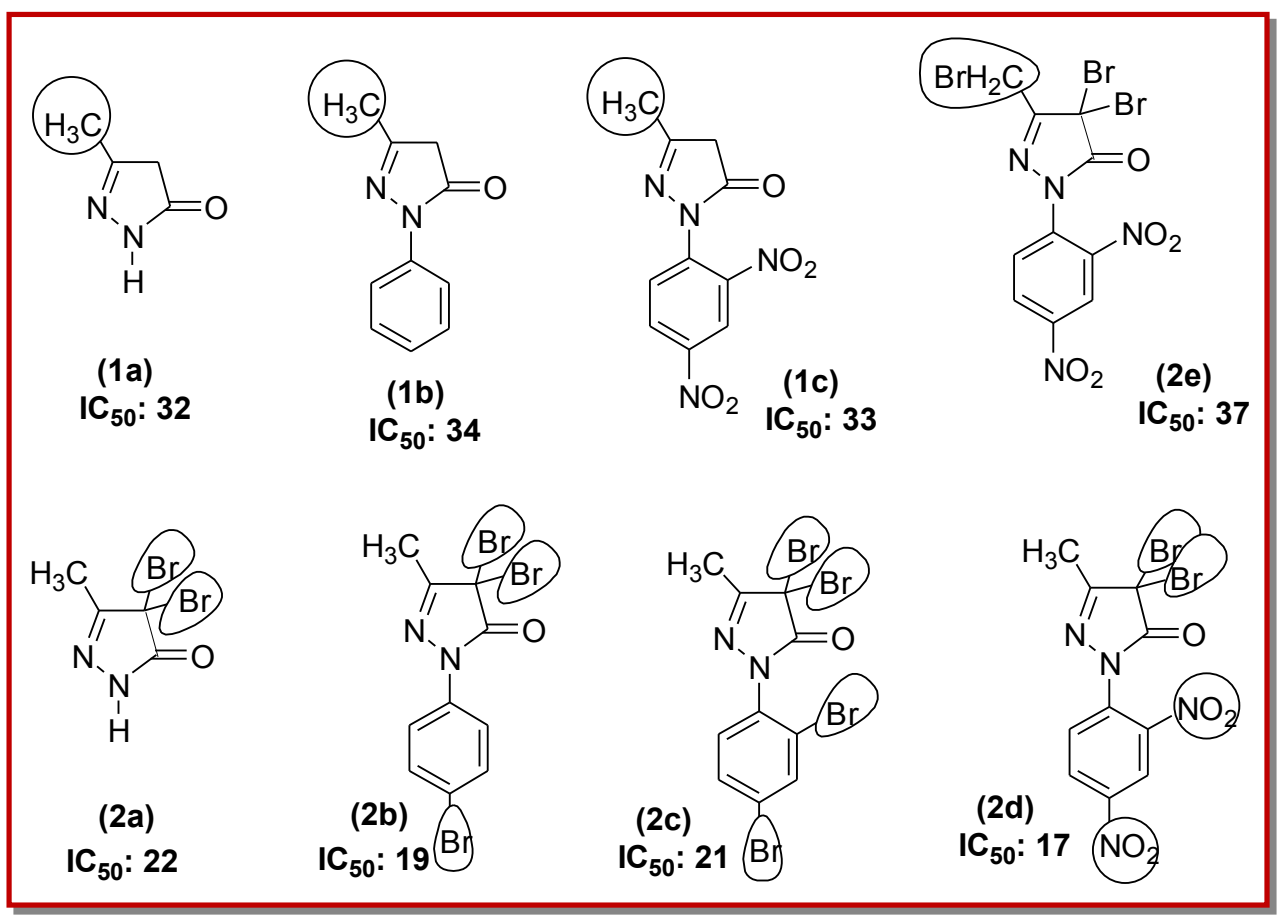

184/186/188 (12.63\%); 183 (52.0\%); 105 (17.23\%); 93 $(17.07 \%)$.

Compound 3: IR (Nujol): 2960, 2873 (v $\mathrm{v}_{\mathrm{C}-\mathrm{H}}$ aliphatic); 1708.5 ( $\mathrm{v}_{\mathrm{C}=\mathrm{O}}$ ketone); 1756.02 ( $\mathrm{v}_{\mathrm{C}=\mathrm{O}}$ ester); $1455,1358\left(\delta_{\mathrm{C}-}\right.$ H); 1015 ( $\left.\mathrm{v}_{\mathrm{C}-\mathrm{O}}\right) ; 742\left(\mathrm{v}_{\mathrm{C}-\mathrm{Br}}\right) ; \mathrm{cm}^{-1} ;{ }^{1} \mathrm{H}-\mathrm{NMR}\left(\mathrm{CDCl}_{3}\right): \delta 2.85$ $(\mathrm{s}, 3 \mathrm{H}) ; 1.36(\mathrm{t}, 3 \mathrm{H}, J=6.89 \mathrm{~Hz}) ; 4.82(\mathrm{q}, 2 \mathrm{H}, \mathrm{J}=6.82 \mathrm{~Hz})$.

The comparative $\mathrm{IC}_{50}$ values of the synthesized compounds are shown bellow.

According to the $\mathrm{IC}_{50}$ values the compounds are remarked as very highly active $\left(\mathrm{IC}_{50}<19\right)$, highly active $\left(19<\mathrm{IC}_{50}<29\right)$, weakly active $\left(29<\mathrm{IC}_{50}<39\right)$, less active $\left(39<\mathrm{IC}_{50}<69\right)$ and inactive $\left(69<\mathrm{IC}_{50}\right)$.

Figure 2 represents the comparative $\mathrm{IC}_{50}$ values of the synthesized compounds (1a, 1b, 1c, 2a, 2b, 2c, 2d, 2d-1, $2 e$ and 3) and Table III indicates the remarks about the cytotoxicity.

\section{Discussion}

After performing the bioassay of the synthesized molecules, it was found that the terminal methyl group is necessary for its activity. When the compounds $\mathbf{1 a}, \mathbf{1 b}$ and 1c were brominated the activity of the molecule was increased. The activity was further increased when two nitro-groups were introduced in the benzene moiety in ortho- and para- position.

Hence the terminal methyl chain should be undisturbed to show fruit-full bioactivity. According to Regan et al. 2003 the terminal small alkyl chains are sterically fit into the lipophilic active pocket of the enzyme. It is also proved by the present work. When the terminal methyl group is disturbed by introducing a bulky group- $\mathrm{Br}$, the activity (hence $\mathrm{IC}_{50}$ ) reduces by 3 to 5 . Thus when the methyl side chain at position 5 was monobrominated along with the bromination (in $2 \mathrm{c}-2$ ) at the active methylene group (position 4), the activity was decreased remarkably. Thus the methyl side chain, brominated active methylene group (position 4) and the two nitro-groups containing phenyl ring in the pyrazolone ring are very important to show biological activity.

The present studies agree with the earlier experiments. Jiang et al. (1990) showed that 2-styrylquinazolin-4(3H)ones have potential antimitotic anticancer activity. Their extensive structure activity relationship (SAR) studies suggested that the entire quinazolinone was required but activity was further enhanced by introducing halide group into the 4 position of pyrazole ring. The present findings are also consistent with this literature. But in the case of compound $2 \mathbf{e}$ in which the methyl side chain is also brominated showed lower activity. On the other hand, Elzein et al., 2004 showed that alkyl group on pyrazole ring substantially increase its activity as selective adenosine $\mathrm{A}(3)$ receptor. Furthermore, Ikeda et al., 1996 and Huang et al., 1992 showed that methyl group is necessary for the binding of drug through the lipophilic domain of the enzymes. Thus the methyl group on 5 position of pyrazolone ring should be unsubstantiated for showing bioactivity.

Besides, extensive SAR studies showed that the 
benzene ring of the 5-methyl-2-phenyl-2,4-dihydropyrazol-3-one should be substituted by $-\mathrm{Br}$ or $-\mathrm{NO}_{2}$ group to show better activity.

\section{Acknowledgement}

We express our gratitude and thanks to Prof. Helmut Duddeck, Institute of Organic Chemistry, Hannover University, Germany for supplying the MS and NMR spectra of the synthesized compounds. We also express our gratitude to Mrs. Rabeya Khatun, Quality Control Manager, Supreme Pharmaceuticals Ltd. for supplying the IR spectra of the synthesized compounds.

\section{References}

Amir M, Kumar H, Khan SA. Synthesis and pharmacological evaluation of pyrazoline derivatives as new antiinflammatory and analgesic agents. Bioorg Med Chem Lett. 2008; 18: 918-22.

Anzai K, Furuse M, Yoshida A, Matsuyama A, Moritake T, Tsuboi K, Ikota N. In vivo radioprotection of mice by 3methyl-1-phenyl-2pyrazolin-5-one (edaravone; Radicut), a clinical drug. J Radiat Res (Tokyo). 2004; 45: 319-23. http:// dx.doi.org/10.1269/jrr.45.319

Bekhit AA, Fahmy HT. Design and synthesis of some substituted 1H-pyrazolyl-oxazolidines or 1H-pyrazolylthiazolidines as anti-inflammatory-antimicrobial agents. Arch Pharm (Weinheim). 2003; 336: 111-18. http:// dx.doi.org/10.1002/ardp.200390007

Dolle RE, Singh J, Rinker J, Hoyer D, Prasad CV, Graybill TL, Salvino JM, Helaszek CT, Miller RE, Ator MA. Aspartyl alpha-[1-phenyl-3-(trifluoromethyl)-pyrazol-5-yl]methyl ketons as interleukin-1 beta converting enzyme inhibitors. Significance of the P1 and P3 amido nitrtogens for enzymepeptide inhibitor binding. J Med Chem. 1994; 37: 3863-66.

http://dx.doi.org/10.1021/jm00049a001 http://dx.doi.org/10.1021/jm00031a003 http://dx.doi.org/10.1021/jm00043a002

Elguero PJ, Guiraud G, Jacquier R. Nouvelles Réactions de bromination en séric pyrazole. Obtention de bromo-4 pyrazolones. Bulletin de la Socété Chimique de France. 1967; 62: 328-32.

Elzein E, Palle V, Wu Y, Maa T, Zeng D, Zablocki J. 2-Pyrazolyl $-\mathrm{N}(6)$-sustituted adenosine derivatives as high affinity and selective adenosine $\mathrm{A}(3)$ receptor agonists. J Med Chem. 2004; 47: 4766-73. http://dx.doi.org/10.1021/jm049682h

Friebolin H. Basic one and two-dimensional NMR Spectroscopy. $3^{\text {rd }}$ ed. Germany, Wiley-VCH, 1998, p 47.

GÜrsoy A, Demiravak S, Capan G, Erol K, Vural K. Synthesis and preliminary evaluation of new 5-pyrazolione derivatives as analgesic agents. Eur J Med Chem. 2000; 35: 359-64. http:/ / dx.doi.org/10.1016/S0223-5234(00)00117-3

Huang LJ, Hour MJ, Teng CM, Kuo SC. Synthesis and antiplatelat activities of N-arylmethyl-3,4-dimethylpyrano [2,3-c]pyrazole-6-one derivatives. Chem Pharm Bull (Tokyo). 1992; 40: 2547-51.

Ikeda M, Maruyama K, Nobuhara Y, Yamada T, Okabe S. Synthesis and cytoprotective antiulcer activity of 2- or 4- $(1 \mathrm{H}-$ pyrazol-1-yl)pyrimidine derivatives related to mepirizole and dulcerozine. Chem Pharm Bull (Tokyo). 1996; 44: 170006.

Islam MR, Khayer KK, Ahmad MM. Synthesis of 3methylpyrazolin-5-one and its 3,3-dimethyl-5,5-ethene-bis-2pyrazolin-4,4-dione, substituted cyclic tri-azathione and Nacetylated derivatives. J Bangladesh Chem Soc. 2001; 14: 3339.

Jiang JB, Hesson DP, Dusak BA, Dexter DL, Kang GI, Hamel E. Synthesis and biological evaluation of 2-styrylquinazolin-4 $(3 \mathrm{H})$-ones, a new class of antimitotic anti-cancer agents which inhibit tubulin polymerization. J Med Chem. 1990; 33: 1721-28. http://dx.doi.org/10.1021/jm00168a029

Kativar SB, Srivastava K, Puri SK, Chauhan PM. Synthesis of 2[3,5-substituted pyrazole-1-yl]-4,6-trisubstituted triazine derivatives as antimalarial agents. Bioorg Med Chem Lett. 2005; 15: 4957-60. http://dx.doi.org/10.1016/ j.bmcl.2005.08.023

Kuettel S, Zambon A, Kaiser M, Brun R, Scapozza L, Perozzo R. Synthesis and evaluation of antiparasitic activities of new 4-[5-(4-phenoxy phenyl)-2H-pyrazol-3-yl]morpholine derivatives. J Med Chem. 2007; 50: 5833-39. http:// dx.doi.org/10.1021/jm700938n

Li J, DeMello KM, Cheng H, Sakya SM, Bronk BS, Rafka RJ, Jaynes BH, Ziegler CB, Kilroy C, Mann DW, Nimz EL, Lynch MP, Haven ML, Kolosko NL, Minich ML, Li C, Dutra JK, Rast B, Crosson RM, Morton BJ, Kirk GW, Callaghan KM, Koss DA, Shavnya A, Lund LA, Seibel SB, Silvia A. Discovery of a potent, selective and orally active canine COX -2 inhibitor, 2-(3-difluoromethyl-5-phenyl-pyrazol-1-yl)-5methane-sulfonyl-pyridine. Bioorg Med Chem Lett. 2004; 14: 95-98. http://dx.doi.org/10.1016/j.bmcl.2003.10.004

Manna F, Chimenti F, Bolasco A, Amico MD, Parrillo C, Rossi F, Marmo E. Anti-inflammatory, analgesic and antipyretic N -acetyl- $\Delta 2$-pyrazolines and dihydro-thienocoumarines. Eur J Med Chem. 1992; 27: 633-39. http:// dx.doi.org/10.1016/0223-5234(92)90142-N

Parmar VS, Kumar A, Prasad AK, Singh SK, Kumar N, Mukherjee S, Raj GH, Goel S, Errington W, Puar MS. Synthesis of E- and Z-pyrazolylacrylonitriles and their evaluation as novel antioxidants. Bioorg Med Chem. 1999; 7: 1425-36. http:// dx.doi.org/10.1016/S0968-0896(99)00056-5

Regan J, Capolino A, Cirillo PF, Gilmore T, Graham AG, 
Hickey E, Kroe RR, Madwed J, Moriak M, Nelson R, Pargellis CA, Swinamer A, Torcellini C, Tsang M, Moss N. Structure activity relationships of the p38alpha MAP kinase inhibitor 1-(5-tert-butyl-2-p-tolyl-2H-pyrazol-3yl)-3-[4-(2morpholin-4-yl-ethoxy) maphthalen-1-yl]urea (BIRB 796). J Med Chem. 2003; 46: 4676-86. http://dx.doi.org/10.1021/ jm030121k

Satyanarayana K, Rao MNA. Synthesis of 4-[5-(substituted
aryl)-4,5-dihydro-1H-pyrazol-3-yl]-3-phenyl-sydnones as anti-inflammatory, antiarthritic and analgesic agents. Eur J Med Chem. 1995; 30: 641-45. http:// dx.doi.org/10.1016/0223-5234(96)88280-8

Solis PN, Wright CW, Anderson MM, Gupta MP, Philipson JD. A microwell cytotoxicity assay using Artemia salina (brine shrimp). Planta Med. 1993; 59: 250-52. http:// dx.doi.org/10.1055/s-2006-959661 